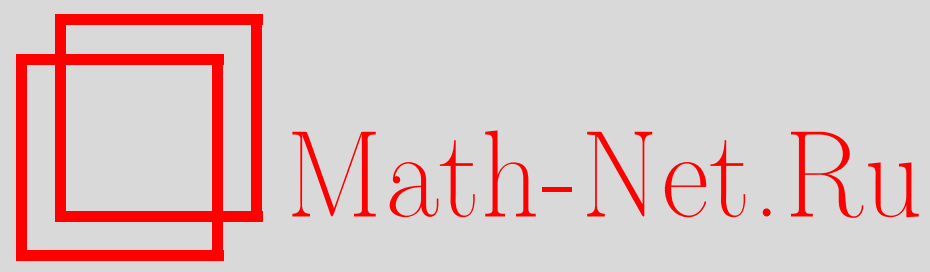

Д. Г. Кореневский, Критерии устойчивости решений систем линейных детерминированных и стохастических разностных уравнений с непрерывным временем и запаздыванием, Матем. заметки, 2001, том 70, выпуск 2, 213-229

DOI: https://doi.org/10.4213/mzm735

Использование Общероссийского математического портала Math-Net.Ru подразумевает, что вы прочитали и согласны с пользовательским соглашением http://www.mathnet.ru/rus/agreement

Параметры загрузки:

IP : 54.209 .52 .79

26 апреля 2023 г., 15:51:33

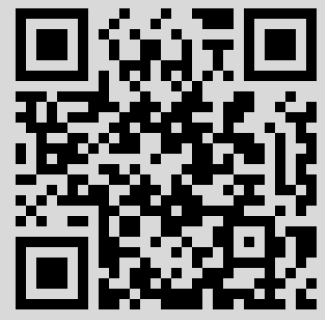




\title{
КРИТЕРИИ УСТОЙЧИВОСТИ РЕШЕНИЙ СИСТЕМ ЛИНЕЙНЫХ ДЕТЕРМИНИРОВАННЫХ И СТОХАСТИЧЕСКИХ РАЗНОСТНЫХ УРАВНЕНИЙ С НЕПРЕРЫВНЫМ ВРЕМЕНЕМ И ЗАПАЗДЫВАНИЕМ
}

\section{Д. Г. Кореневский}

\begin{abstract}
Представлены спектральные и алгебраические коэффициентные критерии (необходимые и достаточные условия), а также достаточные алгебраические коэффициентные условия асимптотической устойчивости по Ляпунову решений указанных в заглавии статьи систем разностных уравнений в случае рационального соотношения между запаздываниями при стохастических возмущениях коэффициентов типа белого шума. Для стохастических систем изучается асимптотическая устойчивость в среднем квадратичном. Используется метод функций Ляпунова. Алгебраические коэффициентные критерии и достаточные условия сформулированы в терминах матричных уравнений Ляпунова (детерминированные системы) и матричных уравнений Сильвестра (стохастические системы).

Библиография: 10 названий.
\end{abstract}

Введение. В этой статье развиваются предыдущие исследования автора по устойчивости по Ляпунову [1], [2], относившиеся к детерминированным и стохастическим системам разностных уравнений с дискретным временем и запаздыванием, на новые классы разностных уравнений - детерминированные и стохастические разностные уравнения с непрерьвньм временем и запаздьванием. В статье получены спектральные и алгебраические коэффициентные критерии, а также достаточные алгебраические коэффициентные условия асимптотической устойчивости решений таких систем разностных уравнений в случае рационального соотношения между запаздьваниями при стохастических возмущениях коэффициентов типа “белого" шума. Для стохастических систем изучается асимптотическая устойчивость в среднем квадратичном. Алгебраические коэффициентные критерии и достаточные условия выражены в терминах матричных уравнений Ляпунова (детерминированные системы) и матричных уравнений Сильвестра (стохастические системы).

Для вывода достаточных условий, как и в случае дискретного времени [1], [2], используются $\left(n^{2}+m\right)$-параметрические функции Ляпунова в виде линейной комбинации квадратичной формы фазовых переменных в текущий момент времени и таких же квадратичных форм с фазовыми переменными в сдвинутые влево по временной оси на величину запаздываний моменты времени ( $n$ - размерность системы, $m$ - количество запаздываний). 
1. Постановка задачи. Рассмотрим начальную задачу для системы линейных детерминированных разностных уравнений с непрерьвньп временем $t$ и постоянными запаздываниями аргумента

$$
y\left(t+\tau_{0}\right)=A_{0} y(t)+\sum_{j=1}^{m} A_{j} y\left(t-\tau_{j}\right), \quad 0 \leqslant t_{0} \leqslant t<\infty,
$$

при начальном условии

$$
y(\theta)=\varphi(\theta), \quad t_{0}-\tau_{m}-\tau_{0} \leqslant \theta \leqslant t_{0},
$$

и начальную задачу для системы линейных стохастических разностных уравнений (которая получается из (1.1) при случайных возмущениях коэффициентов)

$$
x\left(t+\tau_{0}\right)=\left[A_{0}+B_{0} \xi(t)\right] x(t)+\sum_{j=1}^{m}\left[A_{j}+B_{j} \xi(t)\right] x\left(t-\tau_{j}\right)
$$

при том же начальном условии (1.2): $x(\theta)=\varphi(\theta), t_{0}-\tau_{m}-\tau_{0} \leqslant \theta \leqslant t_{0}$.

Здесь вектор-столбцы $y, x \in \mathbb{R}^{n}$; постоянные матрищы коэффищиентов $A_{0}, A_{j}, B_{0}$, $B_{j} \in \mathbb{R}^{n \times n} ; \varphi(\theta)$ - детерминированное начальное условие (непрерывная начальная вектор-функция, $\left.\varphi \in \mathbb{R}^{n}\right) ; \tau_{0}$ - положительная постоянная, шаг итерации; $\tau_{j}(j=1, \ldots, m$; $\left.\tau_{0}<\tau_{1}<\cdots<\tau_{m}\right)$ - положительные постоянные, назьваемые запаздываниями аргумента $t$ и находящиеся в рациональных соотношениях вида $\tau_{j}=j \tau_{0}(j=1, \ldots, m)$; $\xi(t)$ - возмущающий коэффициенты системы (1.1) скалярньй стационарный случайный процесс типа стандартного “белого" шума (так что

$$
\mathrm{M}\{\xi(t)\}=0, \quad \mathrm{M}\left\{\xi(t)^{2}\right\}=1, \quad \mathrm{M}\left\{\xi(t) \xi\left(t_{1}\right), t \neq t_{1}\right\}=0
$$

где М - символ математического ожидания).

ОПРЕДЕЛЕНИЕ 1. Под решением задачи (1.1) понимается $n$-мерная вектор-функция $y\left(t ; t_{0}, \varphi\right)$, обращающая при $t_{0}<t$ уравнение $(1.1)$ в тождество, а при $t \leqslant t_{0}$ совпадающая с $\varphi(\theta)$.

Методом шагов можно установить, что решение $y\left(t ; t_{0}, \varphi\right)$ существует при $t_{0}<t$ и однозначно определяется начальными условиями - моментом времени $t_{0}$ и вектор-функцией $\varphi(\theta)$. При этом решение $y\left(t ; t_{0}, \varphi\right)$ будет непрерывным на интервале $\left[t_{0}-\tau_{m}-\tau_{0}, \infty\right)$ тогда и только тогда, когда непрерывная вектор-функция $\varphi$ удовлетворяет условию

$$
\varphi\left(t_{0}\right)=A \varphi\left(t_{0}-\tau_{0}\right)+\sum_{j=1}^{m} A_{j} \varphi\left(t_{0}-\tau_{j}-\tau_{0}\right) .
$$

Если условие (1.4) не вьполняется, то речь может идти лишь о существовании кусочно-непрерывного решения.

Стачионарным решением (точкой покоя, точкой равновесия) системы разностных уравнений $(1.1)$ называется решение $y\left(t ; t_{0}, \varphi\right)=y_{0}$, равное постоянному вектору $y_{0}$ как на начальном множестве $\left[t_{0}-\tau_{m}-\tau_{0}, t_{0}\right]$, так и при $t_{0}<t$ (аналог дифференциально-разностных уравнений [3, с. 115]); очевидно, для этого необходимо условие (1.4). 
Определение асимптотической устойчивости решений системы разностных уравнений (1.1) вводится по аналогии с определением асимптотической устойчивости решений других функциональных уравнений (дифференциальных, дифференциально-разностных) и содержится, например, в книге [4, с. 111-112].

В пространстве начальных вектор-функций $\varphi$ вводится норма $|\|\varphi \mid\|$ :

$$
\|\varphi\|=\sup _{t_{0}-\tau_{m}-\tau_{0} \leqslant \theta \leqslant t_{0}}\{\|\varphi(\theta)\|\} .
$$

Под знаком супремума стоит обычная евклидова норма вектора.

ОПРЕДЕЛЕниЕ 2. Невозмущенное стационарное (тривиальное) решение $y=0$ системы (1.1) назьвается устойчивым по Ляпунову, если для каждого положительного числа $\varepsilon$ и любого $t_{0} \geqslant 0$ можно указать другое положительное число $\delta=\delta\left(\varepsilon, t_{0}\right)$, зависящее от $\varepsilon$ и $t_{0}$ и такое, что если

$$
\|\varphi\| \mid<\delta\left(\varepsilon, t_{0}\right),
$$

то при всех $0 \leqslant t_{0} \leqslant t$ для возмушенного решения $y\left(t ; t_{0}, \varphi\right)$ выполняется неравенство

$$
\left\|y\left(t ; t_{0}, \varphi\right)\right\|<\varepsilon .
$$

ОПРЕДЕЛЕниЕ 3. Невозмущенное стационарное (тривиальное) решение $y=0$ системы (1.1) назьвается асимптотически устойчивым по Ляпунову, если оно устойчиво в смысле определения 2 и, кроме того, существует предел

$$
\lim _{t \rightarrow \infty}\left\|y\left(t ; t_{0}, \varphi\right)\right\|=0 \text {. }
$$

Аналогично вводится понятие решения системы линейных стохастических разностных уравнений (1.3) с привлечением вероятностных терминов теории случайных процессов .

Под решением начальной задачи (1.3) понимается $n$-мерная случайная векторфункция $x\left(t ; t_{0}, \varphi(\theta)\right)$, которая с вероятностью единица при каждом $t_{0}<t$ обращает уравнение (1.3) в тождество, а при $t_{0}-\tau_{m}-\tau_{0} \leqslant t \leqslant t_{0}$ совпадает с $\varphi(\theta)$.

Существование решения для системы (1.3) при соответствующем начальном условии и указанных выше свойствах начальной вектор-функции также имеет место. Как уже оговаривалось во введении, для стохастической системы (1.3) в дальнейшем рассматривается лишь асимптотическая устойчивость в среднем квадратичном.

ОПРЕДЕЛЕниЕ 4. Невозмущенное стационарное (тривиальное) решение $x=0$ системы (1.3) назьвается устойчивым по Ляпунову в среднем квадратичном, если для каждого положительного числа $\varepsilon$ и любого $0 \leqslant t_{0}$ можно указать другое положительное число $\delta=\delta\left(\varepsilon, t_{0}\right)$, зависящее от $\varepsilon$ и $t_{0}$ и такое, что если

$$
\|\varphi\| \mid<\delta\left(\varepsilon, t_{0}\right)
$$

то при всех $0 \leqslant t_{0} \leqslant t$ для условного математического ожидания квадрата нормы возмущенного решения $x\left(t ; t_{0}, \varphi\right)$ выполняется неравенство

$$
\mathrm{M}\left\{\left\|x\left(t ; t_{0}, \varphi\right)\right\|^{2}|\|\varphi\||<\delta\right\}<\varepsilon .
$$


ОПРЕДЕЛЕниЕ 5. Невозмущенное стационарное (тривиальное) решение $x=0$ системы (1.3) назьвается асимптотически устойчивым по Ляпунову в среднем квадратичном, если оно устойчиво в смысле определения 4 и, кроме того, для условного математического ожидания квадрата нормы возмущенного решения $x\left(t ; t_{0}, \varphi\right)$ существует предел

$$
\lim _{t \rightarrow \infty} \mathrm{M}\left\{\left\|x\left(t ; t_{0}, \varphi\right)\right\|^{2} \mid\|\varphi\|<\delta\right\}=0
$$

ПроблемА. Разработать критерии (необходимые и достаточные условия) и достаточные условия асимптотической устойчивости тривиального решения детерминированной системы (1.1) и асимптотической устойчивости в среднем квадратичном тривиального решения стохастической системы (1.3).

В случае детерминированной системы (1.1) в литературе имеются спектральные критерии асимптотической устойчивости (см., например, Хейл [5, с. 336-341]; Харитонов [6], [7]; Жабко, Харитонов [8, $§ 12])$. Они приводятся ниже в п. 2 и охватьвают общий случай произвольного соотношения между запаздываниями, из-за этого они имеют трансцендентньй вид и поэтому трудно проверяемы. В дополнение к ним нами установлены спектральные и алгебраические коэффициентные критерии и некоторые новые достаточные условия, проверка которых существенно упрощается; правда, охватьвают они лишь случай рационального соотношения между запаздьваниями.

Приведенные ниже в п. 3 критерии и достаточные условия асимптотической устойчивости в среднем квадратичном нам представляются новыми, по крайней мере исследование устойчивости решений стохастических разностных систем (1.3) в работах других авторов нам неизвестно.

\section{2. Критерии и достаточные условия асимптотической устойчивости ре- шений детерминированной системы.}

2.1. Некоторые предварительные факты. По аналогии с общепринятой классификацией систем дифференциально-разностных уравнений [3], [5], [8] систему (1.1) логично отнести к системам нейтрального типа (см. [5, с. 38]).

Одним из непосредственных источников появления разностных уравнений (1.1) являются дифференциально-разностные уравнения и проблемы, связанные с ними; в частности:

1) проблема определения точек покоя дифференциально-разностных уравнений запаздьвающего и нейтрального типов (см. [3, гл. $5, \S 3]$;

2) решение систем вполне интегрируемых дифференциально-разностных уравнений нейтрального типа (см. [9, разд. 3, гл. 1]). Например, дифференциально-разностное уравнение нейтрального типа

$$
\frac{d y(t+1)}{d t}+a \frac{d y(t)}{d t}+b y(t+1)+c y(t)=0
$$

при $c=a b$ является вполне интегрируемым. Оно представляется в форме

$$
\frac{d}{d t}[y(t+1)+a y(t)]+b[y(t+1)+a y(t)]=0,
$$

и его интегрирование приводит к соотношению

$$
y(t+1)+a y(t)=\nu e^{-b t}
$$


где $\nu$ - постоянная интегрирования. Таким образом, интегрирование однородного дифференциально-разностного уравнения (2.1) свелось к решению однопараметрического (параметром служит $\nu$ ) семейства неоднородных разностных уравнений (2.2). В [9] имеются и другие примеры вполне интегрируемых уравнений нейтрального типа.

2.2. Спектральные критерии. Спектральный критерий асимптотической устойчивости. Отыскивая решение уравнения (1.1) в виде экспоненты (т.е. в виде элементарной функции $y=e^{\lambda t} c_{1}$, где $c_{1}$ - постоянный вектор-столбец, $\lambda$ - скаляр, нетрудно установить спектральньй критерий асимптотической устойчивости при фиксированных запаздьваниях: для асимптотической устойчивости тривиального решения $y=0$ системы (1.1) необходимо и достаточно, чтобы вещественные части корней $\lambda$ ее характеристического уравнения

$$
\operatorname{det}\left(E-A_{0} e^{-\lambda \tau_{0}}-\sum_{j=1}^{m} A_{j} e^{-\lambda\left(\tau_{j}+\tau_{0}\right)}\right)=0
$$

удовлетворяли условию $\operatorname{Re} \lambda<0$ (см., например, [5, с. 336-341]; [6], [7]); здесь $E$ - единичная матрища соответствующего размера.

В [5, с. 340-342] установлен также следующий спектральный критерий абсолютной (т.е. равномерной по $\tau_{j}$ ) асимптотической устойчивости для системы (1.1).

СПЕКТРАЛЬНЫЙ КРИТЕРИЙ АБСОЛЮТНОЙ АСИМПТОТИЧЕСКОЙ УСТОЙЧИВОСТИ. Тривиальное решение $y=0$ системы (1.1) асимптотически устойчиво при любых постоянных запаздывания $\tau_{j} \in(0, \infty)$ тогда и только тогда, когда для всех вешественных чисел $\theta_{j}(j=0,1,2, \ldots, m), \theta_{j} \in[0,2 \pi]$, модули всех собственных значений матриць $\sum_{j=0}^{m} e^{i \theta_{j}} A_{j}(i=\sqrt{-1})$ меньше единицы.

Так как критерий асимптотической устойчивости и критерий абсолютной асимптотической устойчивости получены без каких-либо предположений о соотношениях между запаздьваниями $\tau_{j}$, то в общем случае они имеют трансцендентный вид и поэтому трудно проверяемые.

В случае рационального соотношения между запаздываниями вида $\tau_{j}=j \tau_{0}(j=1$, $\ldots, m)$ трансцендентность в определенном смысле исчезает, и тогда можно сформулировать новые спектральные и алгебраические коэффициентные критерии, а также алгебраические коэффициентные достаточные условия асимптотической устойчивости решений системы (1.1). Результаты такого исследования излагаются ниже.

При $\tau_{j}=j \tau_{0}$ уравнение $(2.3)$ с помощью подстановки $e^{-\lambda \tau_{0}}=\mu$ преобразуется в алгебраическое уравнение

$$
\operatorname{det}\left(E-\sum_{j=0}^{m} \mu^{j+1} A_{j}\right)=0
$$

В итоге приходим к следующему утверждению.

ТЕОРемА 1 (первый спектральный критерий асимптотической устойчивости в случае рационального соотношения между запаздываниями). Для асимптотической устойчивости решения $y=0$ системы (1.1) необходимо и достаточно, чтобы модули всех корней $\mu$ алгебраического уравнения (2.4) были больше единицы.

При $\tau_{j}=j \tau_{0}(j=1, \ldots, m)$ исходную $n$-мерную систему разностных уравнений с запаздьванием (1.1) можно свести (путем расширения исходного фазового пространства) 
к эквивалентной $n(m+1)$-мерной системе разностных уравнений без запаздьвания, аналогично тому, как это было оговорено в нашей работе [1] для систем разностных уравнений с дискретным временем и рациональньм соотношением между запаздьваниями (см. также [4, с. 26]). Действительно, введем вектор новых переменных $z(t)$ размерности $n(m+1)$ :

$$
z(t)=\left[\begin{array}{c}
y(t) \\
y\left(t-\tau_{1}\right) \\
\cdots \\
y\left(t-\tau_{m}\right)
\end{array}\right] .
$$

Тогда систему (1.1) с запаздыванием можно записать в виде

$$
z\left(t+\tau_{0}\right)=A z(t)
$$

где под $A$ понимается квадратная матрица размера $n(m+1) \times n(m+1)$

$$
A=\left[\begin{array}{cclcc}
A_{0} & A_{1} & \ldots & A_{m-1} & A_{m} \\
E & 0 & \ldots & 0 & 0 \\
0 & E & \ldots & 0 & 0 \\
\ldots & \ldots & \ldots & \ldots & \ldots \\
0 & 0 & \ldots & E & 0
\end{array}\right] .
$$

ТЕОРемА 2 (второй спектральньй критерий асимптотической устойчивости в случае рационального соотношения между запаздываниями). Для асимптотической устойчивости тривиального решения $z=0$ системы $(2.6)$ ( тотической устойчивости тривиального решения $y=0$ системы (1.1)) необходимо и достаточно, чтобы модули всех собственных значений матрицы $А$ были меньше единицы или, что то жее самое, чтобы матрица $A$ была сходящейся.

ДокАЗАТЕЛЬСтво. Доказательство теоремы осуществляется стандартным для детерминированных стационарных разностных уравнений приемом - построением соответствующего характеристического уравнения. Отыскивая общее решение системы $(2.6)$ в виде показательной функции $z(t)=\lambda^{t} c_{1}$ (т.е. в виде элементарной функции; $c_{1}$ и $\lambda$ - постоянные вектор-столбец и скаляр), приходим к характеристическому уравнению

$$
\operatorname{det}(\mu E-A)=0 \quad\left(\mu=\lambda^{\tau_{0}}\right)
$$

откуда следует справедливость теоремы.

2.3. Алгебраические коэффищиентные критерии и достаточные условия. Алгебраические коэффициентные критерии, если их удалось получить, считаются лучшей конечной формой решения задачи об устойчивости, так как в них наглядно проявляется связь между коэффициентами (параметрами) систем и, тем самьм, обеспечивается эффективное управление степенью устойчивости путем варьирования этой связи. Для систем линейных обыкновенных дифференциальных уравнений и систем линейных разностных уравнений с дискретньм временем примерами алгебраических коэффициентных критериев асимптотической устойчивости являются критерии Рауса-Гурвица, Кона-Шура, критерии, формулируемые в терминах матричных алгебраических уравнений Ляпунова. Аналогичных критериев для систем линейных разностных уравнений с 
непрерьвньм временем (1.1) при произвольном соотношении между запаздываниями не существует.

В случае рационального соотношения между запаздьваниями имеется возможность, исходя из представления системы (1.1) в виде (2.6), сформулировать в терминах матричного алгебраического уравнения Ляпунова следующий критерий.

ТЕОРемА 3 (алгебраический коэффициентный критерий). Для асимптотической устойчивости тривиального решения $y=0$ системы (1.1) необходимо и достаточно, чтобы существовала положительно определенная размера $n(m+1) \times$ $n(m+1)$ матрича $H\left(H=H^{T}>0\right)$ - решение матричного алгебраического уравнения Ляпунова

$$
A^{T} H+H A=-E,
$$

əде $E-$ - едничная матрица размера $n(m+1) \times n(m+1)$.

ДоКАЗАТЕЛЬСтво теоремы 3 осуществляется с помощью $n^{2}(m+1)^{2}$-параметрической функции Ляпунова в виде квадратичной формы $z^{T}(t) H z(t)$, где параметрами служат $n^{2}(m+1)^{2}$ элементов матрицы $H$.

2.4. Достаточные алгебраические коэффишиентные условия. Так как в систему уравнений (1.1) входят матрицы размера $n \times n$, а соответствующее расширенной системе $(2.6)$ матричное уравнение Ляпунова (2.8), дающее критерий асимптотической устойчивости, содержит матришы размера $n(m+1) \times n(m+1)$, то расширение фазовогопространства, следовательно, не самый эффективный и экономичный (в смысле сложности вычислений) способ исследования устойчивости.

Поэтому возникает интерес получить достаточные алгебраические коэффищиентные условия асимптотической устойчивости тривиального решения системы (1.1), представляемые алгебраически через коэффициенты системы и вместе с тем позволяюшие иметь дело непосредственно с описьвающими систему (1.1) матрицами $A_{0}, A_{j}(j=1, \ldots, m)$ и с уравнениями Ляпунова с матричными коэффициентами размера $n \times n$. Решение данной задачи, разумеется, упростит (по сравнению с критерием - теоремой 3) проверку асимптотической устойчивости решений системы (1.1).

Для получения достаточных алгебраических коэффищиентных условий будем использовать метод функций Ллпунова. Построим для системы (1.1) функцию Ляпунова $V(y(t))$ в виде линейной комбинации $m+1$ квадратичных форм переменных $y(t)$ и $y\left(t-\tau_{j}\right) \quad(j=1, \ldots, m)$,

$$
V(y(t))=y^{T}(t) H y(t)+\sum_{j=1}^{m} \gamma_{j} y^{T}\left(t-\tau_{j}\right) H y\left(t-\tau_{j}\right)
$$

с неизвестными пока постоянной положительно определенной матрицей $H \in \mathbb{R}^{n \times n}$ $\left(H=H^{T}>0\right)$ и числами (весовыми коэффициентами) $\gamma_{j}$.

Если нам удастся найти уравнения или неравенства, по которым можно однозначно найти матрицу $H>0$ и числа $\gamma_{j}$, то тем самым выбор функции Ляпунова в форме (2.9) оправдан. Далее в ходе построения будет установлено, что для того, чтобы функция вида (2.9) была положительно определенной на решениях $y(t)$ системы $(1.1)$ и ее первая разность $\Delta V(y(t))=V\left(y\left(t+\tau_{0}\right)\right)-V(y(t))$ на этих решениях была отрицательной величиной (другими словами, чтобы $V$ была функцией Ляпунова для системы (1.1)), 
искомая матрица $H$ должна определяться из матричного дискретного уравнения Ляпунова для матрицы $A_{0}$, а числа $\gamma_{j}$ должны быть положительными и меньше единицы, образуя при этом последовательность

$$
0<\gamma_{m}<\cdots<\gamma_{2}<\gamma_{1}<1
$$

Функция (2.9), как видно из ее структуры, является $\left(n^{2}+m\right)$-параметрической $\left(n^{2}\right.$ элементов матрицы $H$ и числа $\left.\gamma_{j}(j=1, \ldots, m)\right)$.

В соответствии с методом функций Ляпунова для исследования устойчивости вычислим первую разность $\Delta V$ функции $V$ на решениях системы (1.1). Имеем

$$
\begin{aligned}
\{\Delta V(y(t))\}_{(1.1)} \equiv & \left\{V\left(y\left(t+\tau_{0}\right)\right)-V(y(t))\right\}_{(1.1)} \\
= & \left\{y^{T}\left(t+\tau_{0}\right) H y\left(t+\tau_{0}\right)+\sum_{j=1}^{m} \gamma_{j} y^{T}\left(t+\tau_{0}-\tau_{j}\right) H y\left(t+\tau_{0}-\tau_{j}\right)\right. \\
& \left.-y^{T}(t) H y(t)-\sum_{j=1}^{m} \gamma_{j} y^{T}\left(t-\tau_{j}\right) H y\left(t-\tau_{j}\right)\right\}_{(1.1)}
\end{aligned}
$$

Учитывая рациональное соотношение между запаздываниями $\tau_{j}=j \tau_{0}(j=1, \ldots, m)$, равенство (2.11) продолжается следующим образом:

$$
=\left[\begin{array}{c}
y(t) \\
y\left(t-\tau_{1}\right) \\
\cdots \\
y\left(t-\tau_{m}\right)
\end{array}\right]^{T} G\left[\begin{array}{c}
y(t) \\
y\left(t-\tau_{1}\right) \\
\cdots \\
y\left(t-\tau_{m}\right)
\end{array}\right] .
$$

В (2.12) через $G$ обозначена симметричная размера $n(m+1) \times n(m+1)$ блочная матрища

$$
\begin{aligned}
& G=\left[\begin{array}{c|c|c|c}
A_{0}^{T} H A_{0}-\left(1-\gamma_{1}\right) H & A_{0}^{T} H A_{1} & A_{0}^{T} H A_{2} & \ldots \\
A_{1}^{T} H A_{0} & A_{1}^{T} H A_{1}-\left(\gamma_{1}-\gamma_{2}\right) H & A_{1}^{T} H A_{2} & \ldots \\
A_{2}^{T} H A_{0} & A_{2}^{T} H A_{1} & A_{2}^{T} H A_{2}-\left(\gamma_{2}-\gamma_{3}\right) H & \ldots \\
\ldots & \cdots & \ldots & \ldots \\
A_{m-1}^{T} H A_{0} & A_{m-1}^{T} H A_{1} & A_{m-1}^{T} H A_{2} & \ldots \\
A_{m}^{T} H A_{0} & A_{m}^{T} H A_{1} & A_{m}^{T} H A_{2} & \ldots
\end{array}\right. \\
& \left.\mid \begin{array}{c|c}
A_{0}^{T} H A_{m-1} & A_{0}^{T} H A_{m} \\
A_{1}^{T} H A_{m-1} & A_{1}^{T} H A_{m} \\
A_{2}^{T} H A_{m-1} & A_{2}^{T} H A_{m} \\
\cdots & \cdots \\
A_{m-1}^{T} H A_{m-1}^{T}-\left(\gamma_{m-1}-\gamma_{m}\right) H & A_{m-1}^{T} H A_{m} \\
A_{m}^{T} H A_{m-1}^{T} & A_{m}^{T} H A_{m}-\gamma_{m} H
\end{array}\right]
\end{aligned}
$$

Система (1.1) асимптотически устойчива, если вьполняется неравенство $\{\Delta V\}_{(1.1)}$ $<0$, а это возможно лишь тогда, когда квадратичная форма (2.12) отрицательно определенная. В свою очередь, квадратичная форма (2.12) отрицательно определенная лишь в том случае, когда отрицательно определенной является матрица $G\left(G=G^{T}<0\right)$. 
Необходимым условием того, что матрица $G<0$, является отрицательная определенность матричных блоков, стоящих на ее главной диагонали, т.е.

$$
\begin{aligned}
A_{0}^{T} H A_{0}-\left(1-\gamma_{1}\right) H & <0, \\
A_{1}^{T} H A_{1}-\left(\gamma_{1}-\gamma_{2}\right) H & <0, \\
\ldots \ldots \ldots \ldots \ldots \ldots \ldots \ldots \ldots \ldots \ldots & \ldots \ldots \\
A_{m-1}^{T} H A_{m-1}-\left(\gamma_{m-1}-\gamma_{m}\right) H & <0, \\
A_{m}^{T} H A_{m}-\gamma_{m} H & <0 .
\end{aligned}
$$

Так как по определению $H>0$, то из (2.14) следует, что коэффициенты $\gamma_{j}(j=1, \ldots, m)$ должны удовлетворять условию $1-\gamma_{1}>0, \gamma_{1}-\gamma_{2}>0, \ldots, \gamma_{m-1}-\gamma_{m}>0, \gamma_{m}>0$ или, что то же самое, условию (2.10). Далее, симметричная блок-матрица $A_{0}^{T} H A_{0}-$ $\left(1-\gamma_{1}\right) H$, стоящая на главной диагонали матрицы $G$ в левом верхнем углу и соответствующая основной фазовой переменной $y(t)$ системы $(1.1)$, лишь тогда отрицательно определенная, ког да существует положительно определенное решение $H>0$ матричного дискретного уравнения Ляпунова

$$
A_{0}^{T} H A_{0}-\left(1-\gamma_{1}\right) H=-E .
$$

Вид матричного уравнения (2.15) и ограничение на $\gamma_{1}(2.10)$ приводят к заключению, что матрища $A_{0}$ должна быть сходящейся (модули ее собственных значений меньше единицы) с некоторым запасом сходимости $\rho(0<\rho<1)$ [2]; следовательно, $\rho$ должно удовлетворять неравенству

$$
1-\rho^{2}<\gamma_{1}<1
$$

Соотношение (2.15) при выбранном согласно (2.10) $\gamma_{1}$ служит уравнением для определения неизвестной матрицы $H$, входящей в функцию Ляпунова (2.9).

Итак, путем использования функции Ляпунова (2.9) пришли к следующему утверждению.

ТЕОРЕМА 4 (достаточные коэффициентные условия асимптотической устойчивости). Тривиальное решение $y=0$ системы (1.1) асимптотически устойчиво, если выполнены следующие условия:

1) матрииа $A_{0}$ сходящаяся с некоторым запасом сходимости $\rho(0<\rho<1)$;

2) существует полохительно определенное решение $H \in \mathbb{R}^{n \times n}\left(H=H^{T}>0\right)$ матричного дискретного уравнения Ляпунова (2.15), в котором полохительное число $\gamma_{1}$ удовлетворяет условию $1-\rho^{2}<\gamma_{1}<1$;

3) имеет место матричное неравенство $G<0$, в котором последовательность произвольно выбираемых положстельных чисел $\gamma_{j}$ удовлетворяет соотношению $0<\gamma_{m}<\cdots<\gamma_{1}<1$.

АЛГОРИТМ ПРИМЕНЕНИЯ ТЕОРЕМЫ 4. Для проверки асимптотической устойчивости надо провести следующие процедуры:

1) проверяем на сходимость матрицу $A_{0}$ и определяем ее запас сходимости $\rho$;

2) выбираем числа $\gamma_{j}$ согласно соотношению (2.10) и $1-\rho^{2}<\gamma_{1}<1$;

3 ) ищем положительно определенное решение $H$ матричного уравнения Ляпунова $(2.15)$; 
4) в случае положительного ответа на пункты 1)-3) подставляем матрицу $H$ и числа $\gamma_{j}$ в матрицу $G(2.13)$ и проверяем ее на отрицательную определенность; если $G<0$, то решения системы (1.1) асимптотически устойчивы.

ЗАмечАниЕ 1. Расширить в пространстве коэффициентов системы (1.1) область достаточных условий асимптотической устойчивости по сравнению с областью, определяемой теоремой 4, можно было бы, если бы удалось построить функцию $V$, имеющую в своей конструкции более чем $n^{2}+m$ свободньх параметров. Выбор в качестве $V$, например, $n^{2}(m+1)$-параметрической комбинации квадратичных форм

$$
V(y(t))=y^{T}(t) H y(t)+\sum_{j=1}^{m} y^{T}\left(t-\tau_{j}\right) H_{j} y\left(t-\tau_{j}\right)
$$

где $H_{j}$ - неизвестные положительно определенные матрицы, подлежащие определению из системы рекуррентных матричных уравнений

$$
A_{j}^{T} H_{j} A_{j}-H_{j}+H_{j+1}=-E \quad(j=1,2, \ldots, m-1),
$$

существенно увеличивает объем вычислений по сравнению с объемом вычислений в теореме 4 и, кроме того, сопровождается неоднозначностью в определении матриц $H_{j}$.

3. Стохастическая система (1.3). Так как стохастическая система (1.3) является системой с переменными коэффициентами, то построить соответствующее характеристическое уравнение непосредственно для (1.3) и, следовательно, сформулировать, например, спектральный критерий в терминах корней характеристического уравнения не представляется возможным.

Один из путей получения критериев (спектрального и алгебраического коэффициентного) асимптотической устойчивости в среднем квадратичном решений системы разностных уравнений (1.3) с рациональным соотношением между запаздываниями - расширение исходного $n$-мерного фазового пространства и построение уравнений для вторых моментов решений расширенной системы разностных уравнений.

3.1. Эквивалентная система стохастических разностных уравнений без запаздывания и уравнения для вторых моментов. Введем в рассмотрение вектор-столбец новых переменных $u(t)$ размера $n(m+1)$,

$$
u(t)=\left[x(t), x\left(t-\tau_{1}\right), \ldots, x\left(t-\tau_{m}\right)\right]^{T} .
$$

Тогда стохастическую систему с запаздьванием можно записать в виде эквивалентной ей стохастической системы размера $n(m+1)$ без запаздьвания

$$
u\left(t+\tau_{0}\right)=[A+B \xi(t)] u(t),
$$

где под $A$ и $B$ понимаются квадратные размера $n(m+1) \times n(m+1)$ блочные матрицы

$$
A=\left[\begin{array}{ccccc}
A_{0} & A_{1} & \ldots & A_{m-1} & A_{m} \\
E & 0 & \ldots & 0 & 0 \\
0 & E & \ldots & 0 & 0 \\
\ldots & \ldots & \ldots & \ldots & \ldots \\
0 & 0 & \ldots & E & 0
\end{array}\right], \quad B=\left[\begin{array}{cccc}
B_{0} & B_{1} & \ldots & B_{m} \\
0 & 0 & \ldots & 0 \\
0 & 0 & \ldots & 0 \\
\ldots & \ldots & \ldots & \ldots \\
0 & 0 & \ldots & 0
\end{array}\right] .
$$


Обозначим через $Q(t)$ матрицу вторых моментов решений системы (1.3), т.е.

$$
Q(t)=\left[q_{i k}(t)\right]_{i, k=1}^{n(m+1)} \equiv \mathrm{M}\left\{u(t) u^{T}(t)\right\} .
$$

Умножая уравнение (3.1) почленно справа на соответствующее ему транспонированное уравнение

$$
u^{T}\left(t+\tau_{0}\right)=u^{T}(t)\left[A^{T}+B^{T} \xi(t)\right]
$$

и применяя к полученному результату операцию математического ожидания с учетом свойств случайного процесса $\xi(t)$, получим разностное матричное уравнение для матрицы вторых моментов

$$
Q\left(t+\tau_{0}\right)=A Q(t) A^{T}+B Q(t) B^{T}
$$

С помощью кронекерового произведения матриц матричное уравнение (3.3) можно записать в векторно-матричной форме

$$
q\left(t+\tau_{0}\right)=\mathscr{A} q(t)
$$

где через $q(t)$ обозначен $n^{2}(m+1)^{2}$-мерньй вектор-столбец,

$$
q(t)=\left[q_{11}(t), \ldots, q_{1 n}(t), \ldots, q_{n(m+1)}(t), \ldots\right]^{T}
$$

а матрица $\mathscr{A}$ размера $n^{2}(m+1)^{2} \times n^{2}(m+1)^{2}$ имеет вид

$$
\mathscr{A}=A \otimes A+B \otimes B .
$$

3.2. Спектральный критерий. Запись уравнения моментов (3.3) в векторно-матричной форме (3.4) позволяет сформулировать спектральньй критерий асимптотической устойчивости в среднем квадратичном в виде следующего утверждения.

ТЕОРема 5. Для асимптотической устойчивости тривиального решения $q=0$ системы (3.4) (и, следовательно, асимптотической устойчивости в среднем квадратичном тривиального решения $x=0$ системы (1.3)) необходимо и достаточно, чтобы модули всех собственных значений матрицы $\mathscr{A}(3.5)$ были меньше единицы (другими словами, чтобы матрича $\mathscr{A}$ была сходящейся).

ДокАЗАтЕльство. Так как система уравнений для моментов (3.4) является детерминированной, то доказательство теоремы осуществляется стандартньм для детерминированных стационарных разностных уравнений приемом - построением соответствующего характеристического уравнения. Отыскивая общее решение системы (3.4) в виде показательной функции $q(t)=\lambda^{t} c_{1}$ (т.е. в виде элементарной функции; $c_{1}$ и $\lambda$ - векторная и скалярная константы соответственно), приходим к характеристическому уравнению

$$
\operatorname{det}(\mu E-\mathscr{A})=0 \quad\left(\mu=\lambda^{\tau_{0}}\right),
$$

откуда следует справедливость теоремы.

3.3. Алгебраические коэффищиентные критерии. Исходя из стохастической разностной системы уравнений расширенной размерности (3.1), можно предложить следующий критерий. 
ТЕОРемА 6 (первый алгебраический коэффициентный критерий). Для асимптотической устойчивости в среднем квадратичном тривиального решения $u=0$ системь (3.1) (и, следовательно, асимптотической устойчивости в среднем квадратичном тривиального решения $x=0$ системы (1.3)) необходимо и достаточно, чтобы существовала полохстельно определенная матрица $H \in \mathbb{R}^{n(m+1) \times n(m+1)}$ - решение матричного алгебраического уравнения Сильвестра

$$
H-A^{T} H A-B^{T} H B=E .
$$

ДокАЗАТЕльство. Введем в рассмотрение $n^{2}(m+1)^{2}$-параметрическую стохастическую функцию Ляпунова вида квадратичной формы

$$
V(u(t))=u^{T}(t) H u(t), \quad H \in \mathbb{R}^{n(m+1) \times n(m+1)} .
$$

Роль параметров играют $n^{2}(m+1)^{2}$ элементов положительно определенной матрицы $H$ $\left(H=H^{T}>0\right)$, подлежащей определению.

Выполняя присущие методу функций Ляпунова для стохастических разностных уравнений шаги (см. [10, гл. 2]) - выгисление первой разности функции Ляпунова (3.7) на решениях (3.1), т.е.

$$
\{\Delta V(u(t))\}_{(3.1)}=\left\{V\left(u\left(t+\tau_{0}\right)\right)-V(u(t))\right\}_{(3.1)}
$$

и последующее исследование условий, при которых математическое ожидание первой разности отрицательно,

$$
\mathrm{M}\{\Delta V(u(t))\}_{(3.1)}<0,
$$

- приходим к утверждению теоремы.

Запись матричного уравнения для моментов (3.3) в векторно-матричной форме (3.4) позволяет сформулировать еще один алгебраический коэффициентньй критерий асимптотической устойчивости в среднем квадратичном.

ТЕОРемА 7 (второй алгебраический коэффициентньй критерий). Для асимптотической устойчивости тривиального решения $q=0$ системы (3.4) ( тельно, асимптотической устойчивости в среднем квадратичном тривиального решения $x=0$ системы (1.3)) необходимо и достаточно, чтобь существовала полохительно определенная матрица $H \in \mathbb{R}^{n^{2}(m+1)^{2} \times n^{2}(m+1)^{2}}$ - решение дискретного матричного алгебраического уравнения Ляпунова

$$
H-\mathscr{A}^{T} H \mathscr{A}=E \text {. }
$$

ДокАЗАТЕЛЬСТВо теоремы осуществляется с помощью $n^{4}(m+1)^{4}$-параметрической функции Ляпунова вида квадратичной формы

$$
V(q(t))=q^{t}(t) H q(t), \quad H \in \mathbb{R}^{n^{2}(m+1)^{2} \times n^{2}(m+1)^{2}}
$$

стандартными для метода функций Ляпунова вычислениями. 
3.4. Достаточные алгебраические коэффищиентные условия асимптотической устойчивости в среднем квадратичном. Все сказанноев п. 2 о целесообразности поиска достаточных условий асимптотической устойчивости для детерминированной системы (1.1) в то время, когда имеются критерии асимптотической устойчивости, справедливо и в отношении поиска достаточных условий асимптотической устойчивости в среднем квадратичном решений стохастической системы (1.3).

Для получения достаточных условий используем метод функций Ляпунова. Построим для системы (1.3) функцию Ляпунова $V(x(t))$ в виде линейной комбинации $m+1$ квадратичных форм переменных $x(t)$ и $x\left(t-\tau_{j}\right)$,

$$
V(x(t))=x^{T}(t) H x(t)+\sum_{j=1}^{m} \gamma_{j} x^{T}\left(t-\tau_{j}\right) H x\left(t-\tau_{j}\right)
$$

с неизвестными пока постоянной положительно определенной матрицей $H \in \mathbb{R}^{n \times n}$ $\left(H=H^{T}>0\right)$ и числами (весовыми коэффициентами) $\gamma_{j}(j=1, \ldots, m)$. Если нам удастся найти уравнения или неравенства, из которых можно однозначно определить матрицу $H>0$ и числа $\gamma_{j}$, то тем самым выбор функции Ллпунова в форме (3.10) оправдан. Далее в ходепостроения будет установлено, что для того, чтобы функция (3.10) была положительно определенной на решениях $x(t)$ системы (1.3) и математическое ожидание ее первой разности

$$
\Delta V(x(t))=V\left(x\left(t+\tau_{0}\right)\right)-V(x(t))
$$

на этих решениях было отрищательной величиной (другими словами, чтобы $V$ была функцией Ляпунова для системы (1.3)), искомая матрица $H$ должна определяться из уравнения Сильвестра с матричными коэффициентами $A_{0}, B_{0}$, а числа $\gamma_{j}(j=1, \ldots, m)$ должны быть положительными и меньше единицы, образуя при этом последовательность (2.10).

Функция Ляпунова (3.10), как видно из ее структуры, является $\left(n^{2}+m\right)$-параметрической.

В соответствии с методом функций Ллпунова исследования устойчивости в среднем квадратичном решений разностных стохастических уравнений вычислим первую разность $\Delta V$ функции $V(3.10)$ на решениях системы (1.3) и ее математическое ожидание $\mathrm{M}\{\Delta V\}$. Имеем

$$
\begin{aligned}
\{\Delta V(x(t))\}_{(1.3)}= & \left\{V\left(x\left(t+\tau_{0}\right)\right)-V(x(t))\right\}_{(1.3)} \\
= & \left\{x^{T}\left(t+\tau_{0}\right) H x\left(t+\tau_{0}\right)+\sum_{j=1}^{m} \gamma_{j} x^{T}\left(t-(j-1) \tau_{0}\right) H x\left(t-(j-1) \tau_{0}\right)\right. \\
& \left.-x^{T}(t) H x(t)-\sum_{j=1}^{m} \gamma_{j} x^{T}\left(t-j \tau_{0}\right) H x\left(t-j \tau_{0}\right)\right\}_{(1.3)}
\end{aligned}
$$




$$
\begin{aligned}
= & \left\{\left[A_{0}+B_{0} \xi(t)\right] x(t)+\sum_{j=1}^{m}\left[A_{j}+B_{j} \xi(t)\right] x\left(t-j \tau_{0}\right)\right\}^{T} H \\
& \times\left\{\left[A_{0}+B_{0} \xi(t)\right] x(t)+\sum_{j=1}^{m}\left[A_{j}+B_{j} \xi(t)\right] x\left(t-j \tau_{0}\right)\right\} \\
& +\sum_{j=1}^{m} \gamma_{j} x^{T}\left(t-(j-1) \tau_{0}\right) H x\left(t-(j-1) \tau_{0}\right) \\
& -x^{T}(t) H x(t)-\sum_{j=1}^{m} \gamma_{j} x^{T}\left(t-j \tau_{0}\right) H x\left(t-j \tau_{0}\right) \\
= & x^{T}(t)\left\{\left[A_{0}^{T}+B_{0}^{T} \xi(t)\right] H\left[A_{0}+B_{0} \xi(t)\right]-H\right\} x(t) \\
& +x^{T}(t)\left[A_{0}^{T}+B_{0}^{T} \xi(t)\right] H \sum_{j=1}^{m}\left[A_{j}+B_{j} \xi(t)\right] x\left(t-j \tau_{0}\right) \\
& +\sum_{j=1}^{m} x^{T}\left(t-j \tau_{0}\right)\left[A_{j}^{T}+B_{j}^{T} \xi(t)\right] H\left[A_{0}+B_{0} \xi(t)\right] x(t) \\
& +\left\{\sum_{j=1}^{m} x^{T}\left(t-j \tau_{0}\right)\left[A_{j}^{T}+B_{j}^{T} \xi(t)\right]\right\} H\left\{\sum_{j=1}^{m}\left[A_{j}+B_{j} \xi(t)\right] x\left(t-j \tau_{0}\right)\right\} \\
& -\sum_{j=1}^{m} \gamma_{j} x^{T}\left(t-j \tau_{0}\right) H x\left(t-j \tau_{0}\right) \\
& +\sum_{j=1}^{m} \gamma_{j} x^{T}\left(t-(j-1) \tau_{0}\right) H x\left(t-(j-1) \tau_{0}\right) .
\end{aligned}
$$

Тогда для математического ожидания первой разности получаем следующее представление:

$$
\begin{aligned}
\mathrm{M}\{\Delta V(x(t))\}_{(1.3)}= & \mathrm{M}\left\{x^{T}(t)\left(A_{0}^{T} H A_{0}+B_{0}^{T} H B_{0}-H+\gamma_{1} H\right) x(t)\right. \\
& +x^{T}(t) A_{0}^{T} H \sum_{j=1}^{m} A_{j} x\left(t-j \tau_{0}\right)+\sum_{j=1}^{m} x^{T}\left(t-j \tau_{0}\right) A_{j}^{T} H A_{0} x(t) \\
& +\sum_{j=1}^{m-1} x^{T}\left(t-j \tau_{0}\right)\left[A_{j}^{T} H A_{j}+B_{j}^{T} H B_{j}-\left(\gamma_{j}-\gamma_{j+1}\right) H\right] x\left(t-j \tau_{0}\right) \\
& +x^{T}\left(t-m \tau_{0}\right)\left[A_{m}^{T} H A_{m}+B_{m}^{T} H B_{m}-\gamma_{m} H\right] x\left(t-m \tau_{0}\right) \\
& \left.+\sum_{i, j=1 ; i \neq j}^{m} x^{T}\left(t-i \tau_{0}\right) A_{i}^{T} H A_{j} x\left(t-j \tau_{0}\right)\right\} .
\end{aligned}
$$

Выражение, стоящее в фигурных скобках правой части (3.11), можно рассматривать как квадратичную форму переменньх $x(t), x\left(t-\tau_{0}\right), \ldots, x\left(t-m \tau_{0}\right)$. Тогда (3.11) пере- 
пишется в следующем матричном виде:

$$
\mathrm{M}\{\Delta V(x(t))\}_{(1.3)}=\mathrm{M}\left\{\left[\begin{array}{c}
x(t) \\
x\left(t-\tau_{0}\right) \\
\cdots \\
x\left(t-m \tau_{0}\right)
\end{array}\right]^{T} G_{1}\left[\begin{array}{c}
x(t) \\
x\left(t-\tau_{0}\right) \\
\cdots \\
x\left(t-m \tau_{0}\right)
\end{array}\right]\right\}
$$

где через $G_{1}$ обозначена блочная матрица

$$
\begin{aligned}
& G_{1}=\left[\begin{array}{c|c|c}
A_{0}^{T} H A_{0}+B_{0}^{T} H B_{0}-\left(1-\gamma_{1}\right) H & A_{0}^{T} H A_{1} & \cdots \\
A_{1}^{T} H A_{0} & A_{1}^{T} H A_{1}+B_{1}^{T} H B_{1}-\left(\gamma_{1}-\gamma_{2}\right) H & \cdots \\
\cdots & A_{m-1}^{T} H A_{1} & \cdots \\
A_{m-1}^{T} H A_{0} & A_{m}^{T} H A_{1} & \cdots \\
A_{m}^{T} H A_{0} &
\end{array}\right. \\
& \begin{array}{c}
A_{0}^{T} H A_{m-1} \\
A_{1}^{T} H A_{m-1} \\
\cdots \\
A_{m-1}^{T} H A_{m-1}+B_{m-1}^{T} H B_{m-1}-\left(\gamma_{m-1}-\gamma_{m}\right) H \\
A_{m}^{T} H A_{m-1}
\end{array} \\
& \left.\mid \begin{array}{c}
A_{0}^{T} H A_{m} \\
A_{1}^{T} H A_{m} \\
\cdots \\
A_{m-1}^{T} H A_{m} \\
A_{m}^{T} H A_{m}+B_{m}^{T} H B_{m}-\gamma_{m} H
\end{array}\right]
\end{aligned}
$$

Математическое ожидание (3.12) отрицательно лишь в том случае, когда квадратичная форма, стояшая в (3.12) под знаком математического ожидания справа, на решениях $x(t)$ системы (1.3) отрицательно определенная; другими словами, когда отрицательно определена матрица $G_{1}\left(G_{1}=G_{1}^{T}<0\right)$.

Необходимым условием того, что матрища $G_{1}<0$, является отрицательная определенность матричных блоков, стоящих на ее главной диагонали,

$$
\begin{array}{r}
A_{0}^{T} H A_{0}+B_{0}^{T} H B_{0}-\left(1-\gamma_{1}\right) H<0, \\
A_{1}^{T} H A_{1}+B_{1}^{T} H B_{1}-\left(\gamma_{1}-\gamma_{2}\right) H<0, \\
\ldots \ldots \ldots \ldots \ldots \ldots \ldots \ldots \ldots \ldots \ldots \ldots \ldots \ldots \ldots \ldots \ldots \ldots \ldots \ldots \ldots \ldots \\
A_{m-1}^{T} H A_{m-1}+B_{m-1}^{T} H B_{m-1}-\left(\gamma_{m-1}-\gamma_{m}\right) H<0, \\
A_{m}^{T} H A_{m}+B_{m}^{T} H B_{m}-\gamma_{m} H<0 .
\end{array}
$$

Так как по определению $H>0$, то из (3.14) следует, что коэффициенты $\gamma_{j}$ должны удовлетворять условию $1-\gamma_{1}>0, \gamma_{1}-\gamma_{2}>0, \ldots, \gamma_{m-1}-\gamma_{m}>0, \gamma_{m}>0$, т.e. условию (2.10). Далее, симметричная блок-матрица $A_{0}^{T} H A_{0}+B_{0}^{T} H B_{0}-\left(1-\gamma_{1}\right) H$, стоящая на главной диагонали матрицы $G_{1}$ в левом верхнем углу и соответствующая основной фазовой переменной $x(t)$ системы $(1.3)$, лиш тогда отрицательно определенная, когда существует положительно определенное решение $H>0$ матричного уравнения Сильвестра

$$
A_{0}^{T} H A_{0}+B_{0}^{T} H B_{0}-\left(1-\gamma_{1}\right) H=-E .
$$


Вид матричного уравнения (3.15) и ограничение на $\gamma_{1}(2.10)$ приводят к заключению, что матрица $A_{0}$ должна быть сходящейся с некоторым запасом сходимости $\rho(0 \leqslant \rho<1)$ [2]; это приводит к неравенствам $1-\rho^{2}<\gamma_{1}<1$. Соотношение (3.15) при выбранном согласно (2.10) $\gamma_{1}$ служит уравнением для определения неизвестной матрицы $H$, входящей в функцию Ляпунова (3.10).

Итак, путем использования функции Ляпунова (3.10) приходим к следующему утверждению.

ТЕОРема 8 (достаточные алгебраические коэффициентные условия асимптотической устойчивости в среднем квадратичном). Тривиальное решение $x=0$ уравнения (1.3) асимптотически устойчиво в среднем квадратичном, если выполнены следующие условия:

1) матрииа $A_{0}$ сходящаяся с некоторым запасом сходимости $\rho(0<\rho<1)$;

2) существует полохительно определенное решение $H \in \mathbb{R}^{n \times n}\left(H=H^{T}>0\right)$ матричного уравнения Сильвестра (3.15), в котором произвольно выбираемое число $\gamma_{1}$ удовлетворяет условию $1-\rho^{2}<\gamma_{1}<1$;

3) имеет место матричное неравенство $G_{1}<0$, в котором последовательность произвольно выбираемых положительных чисел $\gamma_{1}$ удовлетворяет соотношению $0<\gamma_{m}<\cdots<\gamma_{1}<1$.

ЗАмЕчАниЕ 2. Нетрудно заметить, что при отсутствии случайньх возмущений коэффициентов $\left(B_{j}=0 ; j=0,1, \ldots, m\right)$ из теоремы 8 следуют достаточные условия асимптотической устойчивости для системы детерминированных линейных разностных уравнений (1.1), содержащиеся в теореме 4.

ЗАмЕчАниЕ 3. Нецелесообразность выбора при поиске достаточных условий устойчивости в среднем квадратичном вместо функции $V$ (3.10) комбинации квадратичных форм

$$
V=x^{T}(t) H x(t)+\sum_{j=1}^{m} x^{T}\left(t-\tau_{j}\right) H_{j} x\left(t-\tau_{j}\right),
$$

где $H_{j}$ - неизвестные матрицы, имеет ту же мотивацию, что и в случае детерминированной системы (замечание 1). В стохастическом случае приходим к необходимости решать рекуррентную систему матричных уравнений

$$
A_{j}^{T} H A_{j}+B_{j}^{T} H_{j} B_{j}-H_{j}+H_{j+1}=-E, \quad j=1, \ldots, m-1 .
$$

ЗАмЕЧАнИЕ 4. Из теоремы 4 для детерминированной системы (1.1) и теоремы 8 для стохастической системы (1.3) видно, что главньй вклад в свойство асимптотической устойчивости решений систем (1.1) и (1.3) вносит матрища $A_{0}$, стоящая при основных фазовых переменных $y(t)$ и $x(t)$ соответственно. От нее требуется не просто сходимость, но сходимость с "запасом"; она же определяет через уравнение Ляпунова (2.15) и уравнение Сильвестра (3.15) главные члены $y^{T}(t) H y(t)$ и $x^{T}(t) H x(t)$ функций Ляпунова (2.9) и (3.10) соответственно. Дополнительные исследования показьвают, что с учетом этого обстоятельства области асимптотической устойчивости в пространстве коэффициентов, доставляемые теоремами 4 и 8 , можно расширить, если в системе строгих неравенств (2.14) и (3.14) неравенства, начиная со второго, заменить на нестрогие неравенства $(\leqslant)$ и от матриц $G$ и $G_{1}$ требовать отрицательную определенность только 
на главных фазовых переменных $y(t)$ и $x(t)$ и неположительную определенность на совокупностях фазовых переменных $y(t), y\left(t-\tau_{1}\right), \ldots, y\left(t-\tau_{m}\right)$ и $x(t), x\left(t-\tau_{1}\right), \ldots, x\left(t-\tau_{m}\right)$ соответственно.

\section{СПИСОК ЦИТИРОВАННОЙ ЛИТЕРАТУРЫ}

[1] Кореневский Д. Г.К асимптотической устойчивости решений систем линейных детерминированных и стохастических стационарных разностных уравнений с запаздыванием // Докл. AH CCCP. 1992. Т. 322. № 2. С. 219-223.

[2] Кореневский Д. Г. Алгебраический коэффициентный критерий сходимости "с запасом" (экспоненциальной устойчивости) решений линейных стационарных разностных уравнений // Докл. АН СССР. 1990. Т. 313. №6. С. 1320-1322.

[3] Эльсгольц Л. Э. Введение в теорию дифференциальных уравнений с отклоняющимся аргументом. М.: Наука, 1964.

[4] Жабко А.П., Харитонов В.Л. Методы линейной алгебры в задачах управления. С.-Пб.: С.-Петербургский ун-т, 1993.

[5] Хейл Дж. Теория функционально-дифференциальных уравнений. М.: Мир, 1984.

[6] Харитонов В.Л. Глобальная устойчивость по сдвигам возмущенных систем разностных уравнений // Автоматика (Киев). 1991. № 3. С. 3-8.

[7] Харитонов В. Л. О сохранении свойства глобальной устойчивости по сдвигам при вариациях параметров // Автоматика и телемеханика. 1992. № 5. С. 26-30.

[8] Беллман Р., Кук К. Дифференциально-разностные уравнения. Мир, 1967.

[9] Шарковский А. Н., Майстренко Ю. Л., Романенко Е. Ю. Разностные уравнения и их приложения. Киев: Наукова думка, 1986.

[10] Кореневский Д. Г. Устойчивость динамических систем при случайных возмущениях параметров. Алгебраические критерии. Киев: Наукова думка, 1989. 